\title{
Medical humanities among the healing arts?
}

\section{H M Evans, D Greaves}

\section{Both "arts in health" and medical humanities may have therapeutic roles, and a shared concern with creative imagination in health care}

M edical humanities is still in the first flush of youth-if not infancy-and questions about its nature, scope, and identity continue to occupy those engaged in it. One of these questions concerns its relationship to the "arts in health" movement, and thus to the therapeutic roles for creative and expressive arts in the clinical situation. This relationship is far from clear, and on examination it is a complicated and intriguing one.

In this journal we proposed from the outset a distinction between two contrasting approaches to medical humanities. We suggested the terms "additive" and "integrated" to mark the contrast, arguing thus:

\begin{abstract}
The [additive] is concerned with complementing medical science and technology through the contrasting perspective of the arts and humanities, but without either side impinging on the other. The [integrated] aims to refocus the whole of medicine in relation to an understanding of what it is to be fully human; the reuniting of technical and humanistic knowledge and practice is central to this enterprise.
\end{abstract}

One of us has also suggested that "... the medical arts are essentially an ornament to medicine, whilst the medical humanities are an integral part of it". $^{2}$ If for "medical arts" we read "arts in health" it would seem that arts in health fits well with the additive approach to medical humanities, but has little or nothing to do with the integrated approach. This might tempt us to think of arts in health as a kind of subset of medical humanities.

That simple solution will not, however do. Quite apart from its (diplomatically) rather unhelpful territoriality, it assumes that all medical humanities practices and inquiries are alike, and also that all arts in health practices and inquiries are alike, and that one could predict from the outset what would count as an instance of either. An attractively neat way of distinguishing them might have been to cast medical humanities in a reflective and theoretical role, as it were considering and commentating upon the essentially practical and therapeutic role intended for the arts in health. But as a review of a number of the matters implicit in certain papers in this issue of Medical Humanities shows, practices and inquiries are not always so readily pigeonholed. The arts in health include more than merely adjunct activities coupled to scientific medicine. And medical humanities is not necessarily a detached undertaking with no therapeutic implications of its own. Generally (though we do not suggest that this was their authors' primary intention) these papers imply that an inquiry or undertaking within medical humanities reflection can itself come to have therapeutic potential, albeit indirectly.

Perhaps this should come as no surprise; indeed we should be acutely disappointed if the medical humanities had no therapeutic potential, at least in terms of their contribution to the education and sustenance of the sensitive diagnostic clinician-or, as the Nuffield Trust suggests, to the wellbeing of communities which rely less on health care resources and more on their own senses of identity and culture. ${ }^{3}$ But the discussion in a number of this issue's papers suggests the contribution might be more immediate, although perhaps also more subtle.

We might begin with Luc Michel's examination of the evidentiary basis of surgical practice. ${ }^{4}$ Michel's analysis is an instance of philosophical reasoning applied to the structures of clinical practice, and philosophical reasoning is eminently one of the "arts" of medical humanities. Whether or not Michel's analysis is right in its conclusions, a valid conclusion can proceed only from appropriate reasoning; this could typically consist in the kind of analysis which Michel undertakes. If, then, such analysis went on to influence the practice of surgery (for instance, in terms of the ways in which innovations are incorporated ) and if the influence led to demonstrably improved outcomes, then this would constitute a therapeutic gain achieved in part from the good influence that was contributed by philosophical reasoning.

The question now arises as to whether there is a sharp difference between two kinds of therapeutic effect. Does an individual therapeutic effect stemming directly from the clinical encounter stand wholly distinct from a more general therapeutic effect stemming from interventions in policy or in the education of practitioners? Alternatively, could both be seen on a continuum? Opinions on this may be more a matter of deciding, rather than discovering, how the two undertakings should be viewed. To help in deciding, let us look at some other aspects of the relationship.

In his paper on the respective contributions of "art" and "science" to medical practice, Andrew Warsop argues for the primacy of the art of medicine. ${ }^{5}$ One extrapolation of this is to see the general phenomenon of medical practice as itself a component of the "healing arts" or the "arts in health". Of course it may be objected that the arts referred to in "arts in health" are organised aesthetic (or expressive) undertakings rather than exercises in craft-" "craft" being implicitly the more natural interpretation of "art"in a phrase like "the healing arts". However, to meet this objection we can make the extrapolation more plausible by drawing attention to the aesthetic aspects of clinical performance. Here, a sense of style, of improvisation, responsiveness, and spontaneity, even rhythm, in the clinical encounter can be an important component of securing the patient's confidence and participation, even trust. Additionally it might make the consultation a more enjoyable and committed experience for patient and clinician alike-adding an unexpected dimension, perhaps, from the patient's viewpoint. Could any of these features be clinically significant? Readers may have their own pertinent experiences on which to judge this question-and indeed, substantial papers on the subject would be welcomed for future issues of this journal.

One indication is famously given in Oliver Sacks's autobiographical account of a serious knee injury in $A$ Leg to Stand On. ${ }^{6}$ He recounts how, still somewhat shocked after receiving the injury on a Norwegian mountain, he was uplifted by the impromptu dancing of a Norwegian doctor who had himself suffered the same dislocation injury, and had made a full recovery. This episode has aspects of both performance and humour, and both might be important in inspiring a dejected patient to believe in the possibility of recovery-something that could very 
plausibly be given the label "therapeutic". Here then humanistic, artful, medicine becomes both a healing art and the occasion of a therapeutic art in health.

Some health care interventions have an overtly aesthetic programme or intention, of course. Medard Hilhorst's paper on aesthetic surgery ${ }^{7}$ is ostensibly concerned with the moral responsibilities which arise in connection with an adolescent's medical decision making; but the chosen example, rhinoplasty, is replete with-even grounded uponaesthetic considerations. As Hilhorst sets it out, the moral analysis in such cases must itself rest heavily upon different individuals' fragile and subjective understandings of the nature of "the beautiful". What is at stake is whether an adolescent's present conviction of what constitutes a beautiful nose is either durable or in itself a sufficient reason to pursue plastic surgery. Judgments on either question, however, rely in part upon what we who judge think beautiful, and on whether our own notions of beauty are either durable or in themselves a sufficient reason to constrain an adolescent's choices.

The successful production of a cosmetic surgical change, which satisfies at least someone's conception of improved beauty, looks persuasively like an instance of a therapeutic role for plastic art (in this case, akin to sculpture) in health care. We could even claim the surgery to be itself an instance of representational art, of which painting and sculpture are typical general forms, in so far as a "beautiful" body part-here, a (more) beautiful nose-is represented in the face of someone thought to be lacking an authentically beautiful nose of their own. It also, however, raises the further question of whether judgments about the beautiful belong in either the medical humanities, or the "arts in health", or perhaps in both. Art criticism and appreciation might well be acknowledged as falling within the wider world of the arts. The therapeutic "uses" of literature and poetry include opportunities to read them as well as to attempt to write them oneself. In these ways, judgments about what constitutes (for instance) a beautiful nose, both in terms of crafting the nose itself and in terms of contributing to a view as to what counts as a beautiful nose (or as to whether this particular nose is an aesthetic success, etc) are seen to play their part in the wider healing art of cosmetic surgery.

Next we come to the size of the sphere of action of the arts in health. Does diagnosis-as distinct from treatment modalities-fall within it? General considerations of diagnosis seem clearly to fall within the purview of the medical humanities: part of the purpose of medical humanities education is to enrich the clinician's sensitivities in diagnosis, not least by affirming the broadest range of contributing factors (that is, historical or "existential", as well as biophysical) in disease causation. Why should this be any less true in terms of the arts in health? An intriguing example is discussed in A B Shaw's paper on the causes of Hamlet's fatefully delayed revenge. ${ }^{8}$ Shaw's implicit suggestion is that the dramatic and literary arts can feature in health care not simply therapeutically, but also diagnostically-albeit that this was not necessarily the intention of the author or dramatist. Shaw's case (contested by Pickering ${ }^{9}$ ) is that Hamlet faithfully records phenomena which today can be recognised under categories simply not available to Shakespeare, to his contemporaries, or (therefore) to the protagonists in his famous tragedy. Thus drama specifically presents to us, observationally and in a narrative structure, the symptomatology of depressive illness which we ourselves identify after the event, as it were assigning it retrospectively, to that event. Of course suggestions like this are controversial. Some take exception to the assigning of-for instance, Freudian ideas to literary characters such as Hamlet; Pickering raises this objection among others to Shaw's own account. ${ }^{9}$ The interesting question now arises as to whether it is one thing to assign symptoms retrospectively, and quite another to assign intentions or motives whose content would make little sense without a particular framework of ideas.

At stake here is whether symptoms have a form or appearance independent of the ideas and mental categories of the sufferer. Various writers have argued, persuasively, that this is not always so. ${ }^{10}{ }^{11}$ If they are right, then it might be just as difficult to assign at least some symptoms retrospectively as it is to assign motives or intentions. Such difficulties appear to be less of a problem in retrospectively distinguishing clinical signs, which are tied to the ideas and beliefs of the observer rather than the person observed. So-for instance, Rembrandt's famous paintings of Bathsheba, assuming them to be faithful, can disclose hyperthyroidism or breast cancer to the modern observer whether or not Rembrandt himself would have recognised either in his model, because the morphology of this woman's body can be seen comparably-even if not identically-by both seventeenth century painter and modern day diagnostician, even though they have different ways of being interested in (ostensibly) the same visual phenomena.

Perhaps the main point of interest here, though, is that either signs or-in some cases-symptoms can be distinguished by an observer (dramatist, painter) and successfully conveyed to a later age, regardless of whether the necessary ideas and categories were available at the time of recording. Where the ideas were not available, then what is observed survives to an age (our own) holding the relevant medicodiagnostic categories, only because of the availability of a particular art medium. This then is a diagnostic rather than a therapeutic role for art, and it is explored in the context of philosophical reflection within medical humanities.

The representational and narrative arts can also help develop our contemporary understanding of matters of health and illness. LaCivita Nixon and Roscoe argue that this is importantly the case in our understanding of ageing. ${ }^{12}$ Their case is that the arts offer a sensitive historical or existential alternative to the conventional biomedical model of ageing as an essentially physical phenomenon; ageing is a "multidimensional" phenomenon which cannot fully be captured by a single dimensional model attending only to the physical. As we noted earlier in connection with Luc Michel's paper, the way we analyse this (and its implications for the relation between arts in health and the medical humanities) depends heavily upon the extent to which these broader perceptions can be shown to lead to improvement in patient care. Generally, if the arts lead to a broader understanding of a health affecting process such as ageing-even if primarily in terms of the way ageing is experienced rather than caused-and if, further, this broader understanding leads to an improvement in patient care, then it can be claimed that here is a further instance of an indirectly therapeutic role for the arts. Moreover, because the arts are engaged here reflectively, as part of an analysis of our understanding of ageing, rather than simply undertaken, experimented with, then we can again suggest an indirectly therapeutic role for medical humanities. The point again is that if to reflect upon the arts, rather than to "do" them, is part of exploring medical humanities, then this provides both a measure of continuity between arts in health and medical humanities and also the prospect of a genuine, albeit indirect, therapeutic role for the medical humanities.

Finally let us consider Ståle Frederiksen's discussion of the "invisibility" of diseases. ${ }^{13}$ Frederiksen is interested in the ways in which the boundaries of our ordinary sense perception-especially vision-are extended by medical technology, and pre-eminently by imaging technology. Such technology allows the invisible to become visible, and thus to become present to the diagnostic and therapeutic gaze. As others have noted, this aligns with medicine's intellectual project of making the invisible take priority over the visible in causal and 
explanatory terms. ${ }^{14-16}$ There are intriguing parallels with the arts, most obviously of course the visual arts, but not solely those. The intentional character of seeing (indeed, of all perception) in both producing and viewing (or listening to) art is classically explored by Berger. ${ }^{17}$ The point here is that both medicine's technological programme and the creative and expressive programmes of art involve ways of seeing that are not necessarily familiar to the vision we bring to ordinary daily life; in both, the way of seeing in question involves reordering the world under an intellectual and imaginative programme which has its own distinct purposes. For medicine the purposes are explanatory, diagnostic, therapeutic; for the arts the purposes might be many and varied, though challenge, persuasion, exploration, celebration or altered consciousness can be among them. (That said, an important difference could be that medicine's purposes are usually driven by the instrumental goods of restoring ordinary function or ordinary experience; whereas it is more likely that art's purposes make no reference to being "driven" by anything outside art, and the artist is more concerned to bring about an extraordinary experience, that is, to displace rather than to restore ordinary experience.)

Now art itself makes use of technological means, if we appreciate "technology" sufficiently broadly. The simple pencil or brush are forms of technology; we have lost sight of this fact only because of their sheer ordinariness and familiarity, just as we are unlikely to think any longer of tin openers or doorlocks under the category of "technology", reserving the term these days more for machines of great complexity and hidden workings involving electronics and exotic materials. No doubt technology in this sense has its appeal for artists as well; and certainly cinema and photography as modern art forms involve the overt use of sophisticated imaging technology specifically for the production of expressive effect. Superficially we might suppose that whereas the application of medical technology requires the clinician to engage in cool, restrained interpretation of the evidence of her "externally", technologically, extended senses, the artist knowingly and autonomously extends his senses "internally" and in imagination, beyond the already stretched boundaries available through the tools and technologies of his chosen medium. This is to overlook, however, the important role of the imagination in science, ${ }^{18} 19$ and the extent to which imagination may be important to the clinician seeking a diagnosis through the recognition of key and telling patterns.

Relatedly, the notion of "narrative" is typically assumed to apply to stories of experience, including those stories told through the arts, in sharp distinction from the scientific. As such, "narrative based medicine" is held to consist in clinical practice which (rightly) attends to more than just the features which are picked out by physical measurement alone, including technological measurement. ${ }^{20}$ This is crucially important, of course, for the kind of sensitive diagnosis and management which readers of this journal will wish to affirm. It obscures, however, the extent to which narrative is a feature of science as well, both in terms of the imaginative and committed human agency central to scientific observation and reasoning, ${ }^{21}{ }^{22}$ and in terms of the sequential and directed nature of the "stories" that are inevitably told about even the most reductionist versions of physiological processes. What goes on at the molecular and genetic levels are still events, processes, outcomes, invested with meaning by the teller of the stories and the listener alike, a meaning that may carry the most ecstatic or the most grievous implications for the human being about whom the molecular story is told.

It seems then that in many senses, the use of medical imaging technology to "make diseases visible" involves several features central to expressive or creative art. These include: the notion of a kind of altered gaze, comparable to the aesthetic gaze in that it relies on an intensified and changed way of seeing; the direct engagement of the imagination in interpretation; and the telling of a story disclosed by the medium or the machinery of visualisation, and interpreted by the operator who is at the same time the narrator. Just as intriguing is the fact that both expressive art and medical technology generate new problems for our experience, obliging us (for different reasons, of course) to confront things which we wouldn't otherwise encounter or notice. A painting by Vermeer draws the implications of light itself to our attention; a medical $x$ ray film shows us the interior of our very selves, and the future contents of our own graves. ${ }^{23}$ In this sense of extending our consciousness, medical technology thereby itself becomes a further instance of "art in health".

In summary, then, the matters implicit in a number of the articles in this issue of Medical Humanities prompt us to qualify our understanding of the territorial division between arts in health and the medical humanities as such. Useful though the operational distinction is between the two, there are at least two general reasons for treating that distinction with caution and scruple. These are, first, the extent to which the arts in health are porous, capable of admitting numerous and surprising additional members; and, second, the extent to which medical humanities securesindeed, even aims at-an indirectly therapeutic effect, when the education, reflection, and analysis which are its direct business lead on subsequently to gains in the nature of patient care.

Therefore the lessons we draw from this are twofold: first, the importance of accepting the diversity of approaches not merely between but also within both arts in health and medical humanities; and, second, the importance of a central project implicit in both, namely gaining a better understanding of the role of imagination and creativity in comprehending the relationship between arts and sciences in medicine and health care.

$J$ Med Ethics: Medical Humanities 2002;28:57-60

\section{Authors' affiliations}

H M Evans, Centre for Arts and Humanities in Health and Medicine, University of Durham $14 / 15$ Old Elvet, Durham DH $13 \mathrm{HP}$, UK D Greaves, Centre for Philosophy and Health Care, University of Wales Swansea, Singleton Park, Swansea SA2 8PP, UK

Correspondence to: Martyn Evans; h.m.evans@durham.ac.uk

\section{REFERENCES}

1 Greaves D, Evans M. Medical humanities [editorial]. Journal of Medical Ethics: Medical Humanities 2000;26:1-2

2 Greaves D. The nature and role of medical humanities. In: Evans M, Finlay I, eds. Medical humanities. London: BM Books, 2001: 13-22: see especially 22.

3 Philipp R, Baum M, Mawson A, et al. Humanities in medicine: beyond the millennium. London: The Nuffield Trust, 1999

4 Michel L, Johnson P. Is surgical mystique a myth and double standard the reality? Journal of Medical Ethics: Medical Humanities 2002;28:66-70.

5 Warsop A. Art, science, and the existential focus of clinical medicine. Journal of Medical Ethics: Medical Humanities 2002;28:74-7.

6 Sacks O. A leg to stand on. London: Pan Books, 1986:26

7 Hilhorst MT. Philosophical pitfalls in cosmetic surgery: a case of rhinoplasty during adolescence. Journal of Medical Ethics: Medical Humanities 2002;28:61-5.

8 Shaw AB. Depressive illness delayed Hamlet's revenge. Journal of Medical Ethics: Medical Humanities 2002;28:92-4.

9 Pickering N. Depressive illness delayed Hamlet's revenge: a response. Journal of Medical Ethics: Medical Humanities 2002;28:94-6.

10 Helman C. Culture, health and illness. London: Butterworth-Heinemann, 1985

11 Morris DB. The culture of pain. Berkeley, California: University of California Press, 1991

12 LaCivita Nixon L, Roscoe LA. Through a widening lens: anticipating deep autumn. Journal of Medical Ethics: Medical Humanities 2002;28:82-7.

13 Frederiksen S. Diseases are invisible. Journal of Medical Ethics: Medical Humanities 2002;28:71-3.

14 Good B. Medicine, rationality and experience. Cambridge: Cambridge University Press, 1994.

15 Kevles BH. Naked to the bone: medical imaging in the twentieth century. New Brunswick, New Jersey: Rutgers University Press, 1997. 
16 Evans $M$. The "medical body" as philosophy's arena. Theoretical Medicine 2001;22:17-32

17 Berger J. Ways of seeing. Harmondsworth: Penguin, 1972

18 Downie R. Science and the imagination in the age of reason. Journal of Medical Ethics: Medical Humanities 2001 ;27:58-63.
19 Pickering NJ. Metaphors and models in medicine. Theoretical Medicine and Bioethics 1999;20:361-75

20 Greenhalgh T, Hurwitz B. Narrative-based medicine. London: BM Books, 1998.

21 Polanyi M. Personal knowledge: towards a post-critical philosophy. Chicago: University of Chicago Press, 1974.
22 Toulmin S. Knowledge and art in the practice of medicine: clinical judgement and historical reconstruction. In: Delkeskamp-Hayes C, Gardell Cutter MA, eds. Science, technology and the art of medicine. Dordrecht: Kluwer Academic Publishers, 1993: 231-49.

23 Mann T. The magic mountain. London: Vintage Books, 1927: see especially 218.

\section{Have your say}

\section{eLetters}

If you wish to comment on any article published in Medical Humanities you can send an eLetter using the eLetters link at the beginning of each article. Your response will be posted on

Medical Humanities online within a few days of receipt (subject to editorial screening).

www.medicalhumanities.com 